\section{A Rapid Screening Approach to Identify Resistance to Basil Downy Mildew (Peronospora belbahrii)}

\author{
Robert M. Pyne \\ New Use Agriculture and Natural Plant Products Program, Department of \\ Plant Biology and Pathology, Rutgers University, 59 Dudley, Road, New \\ Brunswick, NJ 08901
}

Adolfina R. Koroch

The City University of New York, Borough of Manhattan Community College, 199 Chambers Street, Science Department New York, NY 10007

Christian A. Wyenandt

Department of Plant Biology and Pathology, Rutgers University, Rutgers, Agricultural Research and Extension Center, 121 Northville Road, Bridgeton, NJ 08302

\section{James E. Simon ${ }^{1}$ \\ New Use Agriculture and Natural Plant Products Program (NUANPP), Department of Plant Biology and Pathology, Rutgers University, 59 Dudley, Road, New Brunswick, NJ 08901}

Additional index words. cotyledon inoculation, genetic resistance, leaf maturity, Ocimum spp., plant breeding

Abstract. Sweet basil (Ocimum basilicum L.) is among the most widely popular and economically important culinary herbs. Worldwide production of sweet basil has been threatened by a newly emerging disease, downy mildew (Peronospora belbahrii). Although tolerance and resistance have been identified in other Ocimum species, the traditional sweet basils all have been reported to be highly susceptible. There is an urgent need for evaluation of basil germplasm to identify sources of host resistance to $P$. belbahrii within Ocimum spp. and especially among $O$. basilicum species. In searching for genetic resistance, we developed a rapid approach to screen and evaluate downy mildew response at the cotyledon and true leaf growth stages under controlled environmental conditions. To confirm the reliability and reproducibility of this screening method, an experiment was conducted in which three basil species (Ocimum basilicum, sensitive; $O$. xcitriodorum, tolerant; and $O$. americanum, resistant to basil downy mildew) were evaluated for response to downy mildew inoculations at three growth stages. Disease incidence (DI) at the cotyledon growth stage was equal to or greater than true leaf growth stages for all species indicating that cotyledon response to downy mildew inoculations is a viable marker for predicting true leaf stage resistance. This approach was then used to screen 36 USDA-NPGS $O$. basilicum accessions at cotyledon and first true leaf growth stages to identify promising downy mildew-resistant breeding lines. Thirty accessions were susceptible at both growth stages $(D I=1.0)$. Four accessions exhibited little or no sporulation at either growth stage (DI less than 0.06), three of which showed other symptoms including chlorosis and necrosis. One accession, PI 652053, demonstrated no signs or symptoms but differed greatly from other accessions in regard to leaf morphology and habit. Results show that a resistant, mature plant can be identified at the cotyledon growth stage, providing a robust, low-input approach to identify promising downy mildew-resistant breeding material. Field evaluations of basils under high downy mildew pressure confirmed the applicability of this new screening approach to identify resistance to basil downy mildew.

Received for publication 10 Mar. 2014. Accepted for publication 4 Apr. 2014

Funds for this research were provided by USDA/SCRI grant \# 2011-51181-30646.

We thank our collaborators at University of Florida, University of Massachusetts, Cornell University, and those U.S. commercial basil growers and basil buyers for their support and assistance.

${ }^{1}$ To whom reprint requests should be addressed; e-mail jimsimon@rci.rutgers.edu.
Basil (Ocimum spp.) is a highly diverse genus that exhibits wide inter- and intraspecific variation in morphology, genetics, and composition of essential oil (Paton and Putievsky, 1996; Vieira et al., 2003). Sweet basil $(O$. basilicum) is the most economically important basil species in the United States, popularized by demand in the fragrance, flavor, fresh culinary, and dried herb indus- tries (Simon et al., 1990, 1999). Commercial sweet basil is characterized by a specific phenotype of either "large leaf" or "Genovese"type leaf morphology and an essential oil profile that includes the principle phenylpropanoid and terpenoid components linalool, methyl chavicol, eugenol, and 1,8 cineole (De Masi et al., 2006; Vieira and Simon, 2006).

Global production of sweet basil is being threatened by a newly emerging disease, basil downy mildew caused by the obligate oomycete Peronospora belbahrii (Belbahri et al., 2005; Thines et al., 2009). Ten years after being reported in Europe (Garibaldi et al., 2004b) and 5 years since reaching the United States (Blomquist et al., 2009; Roberts et al., 2009), this disease now threatens basil crop production worldwide (Kanetic and Vasiliou, 2014; Martinez-de la Parte et al., 2009; McLeod et al., 2006; Nagy and Horvath, 2011). The initial disease symptom is usually interveinal chlorosis observed on the adaxial leaf surface, which can be ambiguous and easily mistaken for nutrient deficiency allowing the pathogen to persist under the guise of abiotic stress (McGrath et al., 2010). Given mild temperatures and extended periods of leaf wetness (Garibaldi et al., 2007), sporangiophores emerge from stomata within $7 \mathrm{~d}$ of inoculation and accumulate in a purplish-dark gray mass on the abaxial leaf surface (Koroch et al., 2013). The effectiveness and availability of chemical controls listed for basil downy mildew are limited (Gilardi et al., 2013; Homa et al., 2014; Mersha et al., 2012), require intense management and may not be economically feasible for growers. Repeated use of particular chemical control methods risk evolved pathogen resistance (Cohen et al., 2013) and the presence of unwanted chemical residue on the leaf surface when applied as a foliar application (Gilardi et al., 2013).

Multiple basil (Ocimum spp.) varieties were previously reported as potential sources of resistance or tolerance to basil downy mildew (BDM) in a 2009 field trial (Wyenand et al., 2010). That study was the first to report widespread differences in susceptibility in different sweet basil ( $O$. basilicum) varieties/ breeding lines and identify a higher degree of tolerance to BDM in O. xcitriodorum (lemon basil) and resistance in $O$. americanum 'Spice' basil. These species are highly divergent from common sweet basil (O. basilicum), differing greatly in phenotype, secondary metabolite composition (Vieira and Simon, 2006), and genomic constitution (Carovic-Stanko et al., 2010; Koroch et al., 2010). Interspecific breeding of basil species can be hindered by reproductive barriers such as hybrid sterility (Putievsky et al., 1999). Thus, further evaluation of the Ocimum genus, especially within the $O$. basilicum species, is needed to identify more closely related candidates for introgression of downy mildew resistance into common sweet basil (O. basilicum).

Screening for disease resistance in the field is effective but also highly demanding in terms of acreage, labor, fertilizer, and irrigation requirements. Additionally, sweet basil requires 150 to $180 \mathrm{~d}$ after planting to 
reach full maturity (Putievsky and Galambosi, 1999) and is chilling-sensitive, which limits the number of field evaluations possible in temperate, northern regions of the United States. Evaluating seedlings for downy mildew resistance at the first leaf (cotyledon) growth stage is highly desirable because it allows for the rapid screening of many genotypes and conservation of resources necessary when screening mature plants. However, this approach is only effective when it is demonstrated that mature plants maintain or increase disease tolerance relative to younger growth stages (Wang et al., 2000). The relationship between cotyledon and true leaf growth stages in response to downy mildew has been explored in dif(Coelho et al., 2009; Kim et al., 1989; Leckie et al., 1996; Monteiro et al., 2005; Silue et al., 1996). To be an effective approach to screening for BDM resistance in basil, the interaction between growth stage and disease response must first be evaluated and understood. The objective of this study was to determine whether screening basil seedlings for resistance to downy mildew at the cotyledon growth stage is a reliable approach for identification of resistant genotypes at mature plant growth stages.

\section{Materials and Methods}

In this study, three basil species were evaluated for their response to BDM infection at the cotyledon and true leaf stages. Ocimum basilicum 'DiGenova' (DG, sensitive to BDM), Ocimum xcitriodorum 'Sweet Dani Lemon Basil' (SD, tolerant to BDM), and $O$. americanum 'Spice' (SPI, resistant to BDM) were selected based on their varying responses to downy mildew in field evaluations (Wyenandt et al., 2010) (Table 1). An additional $36 O$. basilicum accessions, representing a wide geographic distribution, were obtained from the U.S. Department of Agriculture (USDA) National Plant Germplasm System (NPGS) and evaluated for response to $P$. belbahrii infection at the cotyledon and first true leaf set growth stages. Seed for all treatments were sown in soilless media, Fafard Growing Mix 2 (Sun Gro Horticulture, Agawam, MA), and placed under intermittent mist in Rutgers University greenhouses until emergence of cotyledons from the soil surface. Seedlings were thinned to one plant per cell in a 72-cell (first experiment) or 128-cell (second experiment) flats.

Basil downy mildew inoculum (sporangia) was obtained from a 2011 field trial at the Rutgers Agricultural Research and Exferent species with contradictory results

tension Center in Bridgeton, $\mathrm{NJ}$, and used to inoculate susceptible $O$. basilicum 'DiGenova' stock plants. Identification of $P$. belbahrii as the causal agent for disease signs and symptoms was verified by polymerase chain reaction using specific primer sets previously described (Belbahri et al., 2005). Stock inoculum was maintained on susceptible host plants in an isolated room within the Rutgers University research greenhouse. Sporangia solutions for the experiments in this study were prepared by agitating freshly sporulating leaves of stock plants in distilled water for 5 min. This solution was filtered through a $40-\mu \mathrm{m}$ nylon mesh cell strainer (Thermo Fisher Scientific, Bridgewater, NJ) and centrifuged at $3000 \mathrm{~g}$ for $10 \mathrm{~min}$. The supernatant was discarded and the pellet resuspended in a known volume of distilled water. A Reichert Bright-Line Haemocytometer (Hausser Scientific, Horsham, PA) was used to adjust the final solution concentration as needed for each experiment.

Two chambers were constructed and installed in a single greenhouse room to provide the necessary conditions for $P$. belbahrii infection of inoculated materials. A dew chamber was constructed in which $100 \%$ relative humidity and leaf wetness were maintained by two Trion $707 \mathrm{U}$ Series atomizing humidifiers (Trion Air Purification Systems, Sanford, NC) on opposite ends of the enclosure (Fig. 1). A mist chamber, consisting of a partial enclosure with an opening at the base, was constructed and overhead irrigation was applied every $2 \mathrm{~h}$ for $5 \mathrm{~min}$ during the light cycle only. Temperature settings were set to $20 / 24{ }^{\circ} \mathrm{C}$ corresponding to a $12-\mathrm{h} / 12-\mathrm{h}$ light/dark cycle in which supplemental light was provided only when photosynthetically active radiation fell below $300 \mu \mathrm{E} \cdot \mathrm{m}^{-2} \cdot \mathrm{s}^{-1}$.

Expt. 1. In the first experiment, DG, SD, and SPI seedlings were evaluated for presence of sporulation at the cotyledon, first, and second true leaf set growth stages. Every basil species-growth stage combination formed an independent treatment consisting of 12 plants per experimental unit in a randomized complete block design. Seven-d-old plants (cotyledon growth stage) were inoculated by pipetting $20-\mu \mathrm{L}$ droplets of $5 \times 10^{4}$ sporangia/mL onto the adaxial surface of each cotyledon ( $40 \mu \mathrm{L} /$ plant). Fourteen-d-old (first true leaf pair growth stage) and 21-d-old (second true leaf pair growth stage) plants were inoculated by saturating leaves with $1 \times$ $10^{4}$ sporangia $/ \mathrm{mL}$ solution $(\approx 2 \mathrm{~mL}$ per seedling) using a handheld sprayer. Inoculated seedlings were immediately transferred to the dew chamber for $48 \mathrm{~h}$, then removed and placed in the mist chamber to allow for periods

Table 1. Commercial basil varieties evaluated in Expt. 1.

\begin{tabular}{|c|c|c|c|c|}
\hline Plant ID & $\begin{array}{c}\text { Scientific } \\
\text { name }\end{array}$ & Cultivar & Source & $\begin{array}{c}\text { Field disease } \\
\text { evaluation }\end{array}$ \\
\hline$\overline{\mathrm{DG}}$ & Ocimum basilicum & DiGenova & Stokes & Highly susceptible \\
\hline SD & Ocimum $\times$ citriodorum & $\begin{array}{l}\text { Sweet Dani Lemon } \\
\text { Basil }\end{array}$ & $\begin{array}{l}\text { Johnny's Selected } \\
\text { Seeds }\end{array}$ & Tolerant \\
\hline SPI & Ocimum americanum & Spice & Richters Herbs & Resistant \\
\hline
\end{tabular}

of leaf dryness and emergence of sporangiophores from the leaf surface. A control was administered using distilled water and maintained in separated chambers to account for potential seedborne infection. Seedlings were evaluated for disease susceptibility 7 and $15 \mathrm{~d}$ post-inoculation (DPI). This experiment was repeated three times and data were analyzed according to the Kruskal-Wallis test (SAS Version 9.4; SAS Institute Inc., Cary, NC).

Expt. 2. In a second experiment, the cotyledon and first true leaf pair growth stages were screened on the same plants of 36 USDA-GRIN $O$. basilicum accessions. Treatments consisted of 16 plants per accession growth stage combination and DG, which was included as a susceptible control or negative control. In the first experiment, DG and SD plants inoculated at Day 7 often failed to produce true leaves as a result of the intensity of infection and environmental conditions in the dew and mist chambers. To allow seedlings to form true leaves, cotyledons of 10-d-old seedlings were drop inoculated with $20-\mu \mathrm{L}$ droplets of $5 \times 10^{4}$ sporangia $/ \mathrm{mL}$ as described in Expt. 1 and scored 10 DPI. Seedlings were removed from the mist chamber and grown out to the first true leaf pair growth stage. Plants were then sprayinoculated with a $1 \times 10^{4}$ sporangia $/ \mathrm{mL}$ solution $(\approx 2 \mathrm{~mL}$ per seedling $)$ and placed in the dew chamber. After $48 \mathrm{~h}$, seedlings were moved to the mist bench and true leaves evaluated for disease response as per the first experiment. Temperature, light, and overhead irrigation settings were identical to the previous experiment.

To allow for simple and rapid scoring of many seedlings for response to downy mildew, a binary scale was used in which $0=$ resistant (sporulation was not visible on abaxial leaf surface) or 1 = susceptible (sporulation was visible on abaxial leaf surface). Leaves exhibiting chlorosis in the absence of observable sporulation were scored using a microscopic staining method previously described (Koroch et al., 2013). Plants in which sporangiophores were observed exiting stomata were considered susceptible for either cotyledon or true leaf growth stages. In both experiments, scoring generated a proportion of infected seedlings or disease incidence (DI) for each treatment group (Table 2).

\section{Results and Discussion}

Variation in response to inoculation was observed among the three basil species evaluated in the first experiment (Table 2). True leaves and cotyledons of all plants were scored 7 and 15 DPI representing early and a later stage of disease progress. The SPI displayed no visible sporulation across experiments, whereas all three growth stages of DG were completely infected 15 DPI with the exception of one seedling that became infected 17 DPI. Susceptibility of all growth stages in DG among repeated experiments provided evidence that appropriate environmental conditions and pathogen virulence were present. In comparison, SD demonstrated 


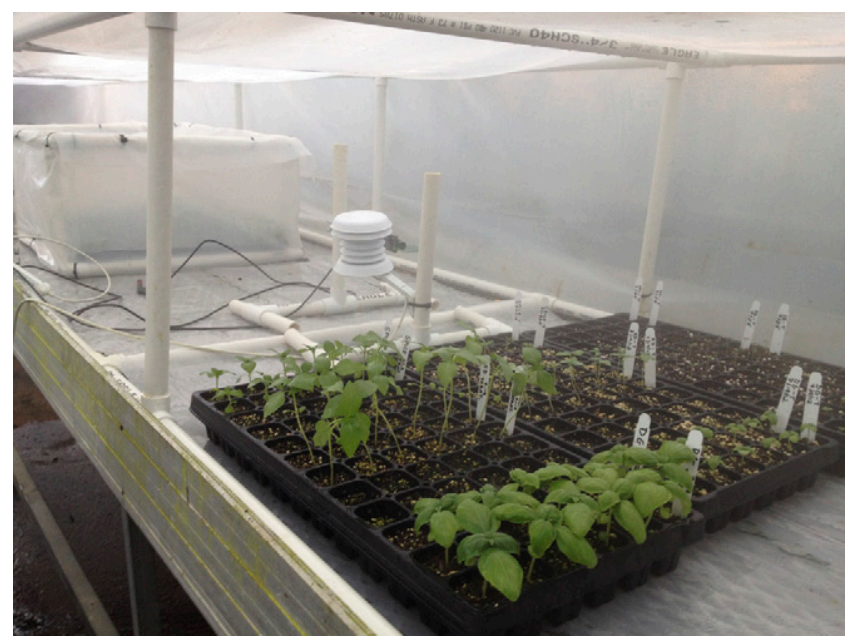

Fig. 1. Dew chamber constructed to provide the necessary conditions for Peronospora belbahrii infection of inoculated materials. Relative humidity and leaf wetness was maintained at $100 \%$ by two Trion $707 \mathrm{U}$ Series atomizing humidifiers (Trion Air Purification Systems, Sanford, NC) on opposite ends of the enclosure. Basil seedlings shown were artificially inoculated with Peronospora belbahrii in Expt. 1.

Table 2. Variation of response by basils to basil downy mildew at cotyledon stage, first, and second true leaf pair at 7 and $15 \mathrm{~d}$ post-inoculation (DPI), Expt. 2.

\begin{tabular}{|c|c|c|c|}
\hline \multirow[b]{2}{*}{ Cultivar ${ }^{y}$} & \multirow[b]{2}{*}{ Growth stage } & \multicolumn{2}{|c|}{ Disease incidence $^{z}$} \\
\hline & & $7 \mathrm{DPI}$ & $15 \mathrm{DPI}$ \\
\hline \multirow[t]{3}{*}{$\overline{\mathrm{DG}}$} & Cotyledon & $0.89 \pm 0.04^{\mathrm{x}}$ & $1.00 \pm 0.00$ \\
\hline & First true leaf pair & $0.72 \pm 0.07$ & $0.97 \pm 0.01$ \\
\hline & Second true leaf pair & $0.81 \pm 0.05$ & $1.00 \pm 0.00$ \\
\hline \multirow[t]{3}{*}{ SD } & Cotyledon & $0.69 \pm 0.06$ & $0.94 \pm 0.03$ \\
\hline & First true leaf pair & $0.53 \pm 0.13$ & $0.61 \pm 0.0$ \\
\hline & Second true leaf pair & $0.00 \pm 0.00$ & $0.00 \pm 0.00$ \\
\hline \multirow[t]{3}{*}{ SPI } & Cotyledon & $0.00 \pm 0.00$ & $0.00 \pm 0.0 c$ \\
\hline & First true leaf pair & $0.00 \pm 0.00$ & $0.00 \pm 0.0 c$ \\
\hline & Second true leaf pair & $0.00 \pm 0.00$ & $0.00 \pm 0.0$ \\
\hline
\end{tabular}

${ }^{\mathrm{z}}$ Mean ratings for downy mildew incidence using a binary scale in which 0 equaled no visible sporulation and 1 equaled visible sporulation on the abaxial leaf surface.

${ }^{\mathrm{y}} \mathrm{DG}=$ O. basilicum 'DiGenova'; $\mathrm{SD}=$ O. xcitriodorum 'Sweet Dani Lemon Basil'; $\mathrm{SPI}=$ O. americanum 'Spice'. No significant difference among growth stages across all cultivars according to the Kruskal-Wallis test $\left(\mathrm{df}=2, \chi^{2}=0.318, P=0.853\right)$.

${ }^{\mathrm{x}} \mathrm{SE}$ among three repeated experiments.

a decreasing mean proportion of infected seedlings relative to increased plant maturity (Table 2), suggesting a developmentally acquired response to infection. The SD cotyledons were susceptible, whereas the second true leaf pair exhibited no infection and the first true leaf pair growth stage produced variable results $(0.61 \pm 0.0715 \mathrm{DPI})$. Differences in responses to downy mildew as a function of leaf maturity and position have been reported in several Brassica spp. (Dickson and Petzoldt, 1993; Jensen et al., 1999; McDowell et al., 2005; Monteiro et al., 2005). In SD, the first true leaf pair growth stage appears to represent a physiological juncture in which the plant begins to demonstrate a host defense response to infection. This acquisition also appears to occur within a relatively short interval between the cotyledon and second true leaf pair and may have varied among seedlings in the SD first true leaf pair growth stage as a result of differences in individual plant growth and maturity.

Expt. 1 can be summarized by three distinct responses to downy mildew among basil species with respect to age: 1) cotyledon and true leaf susceptibility (DG); 2) cotyledon and true leaf resistance (SPI); and 3) cotyledon susceptibility and true leaf resistance (SD). The only combination of responses not observed was cotyledon resistance and true leaf susceptibility. Absence of this response in three divergent basil species demonstrates that true leaf susceptibility does not occur in genotypes with cotyledon stage resistance. These results are in agreement with previous findings in which it was determined that the screening of Brassica oleraceae cotyledons for resistance to downy mildew (Peronospora parasitica) was an effective approach for selection of mature resistant plants because susceptibility of cotyledons was consistently higher or equal to true leaves (Jensen et al., 1999; Wang et al., 2000). Response to downy mildew among genotypes evaluated in this study is consistent with a previous report (Wyenandt et al., 2010) as well as follow-up field trials, which we conducted in 2012 and 2013 (unpublished) in southern and northern New Jersey.
An important characteristic of $P$. belbahrii is the capacity to persist either in or on seed (Farahani-Kofoet et al., 2012; Garibaldi et al., 2004a) providing the opportunity for dissemination and early infection through contaminated seed. Basil genotypes susceptible at early growth stages such as SD have the potential to act as a carrier and distributor of the pathogen as seed or young seedlings (cotyledon and first true leaf growth stages). Evaluation based solely on mature plants ignores the potential for downy mildew susceptibility as transplants.

In the second experiment of this study, 30 of 36 O. basilicum USDA-NGPS accessions exhibited sporulation on all plants $(\mathrm{DI}=$ 1.0) evaluated at cotyledon and true leaf growth stages (Table 3) and were considered susceptible. No sporulation was observed at both growth stages for three accessions and a fourth in which one plant was susceptible $(\mathrm{DI}=0.06)($ Table 3$)$. The USDA lines PI 172996, 172997, and 172998 were considered potential sources of resistance; however, true leaves exhibited chlorosis and necrosis, suggesting a hypersensitive response. Despite this putative defense response in preventing sporulation, disease symptoms of the leaf limit the breeding potential for fresh and culinary basils. In contrast, PI 652053 displayed no signs or symptoms of basil downy mildew, but the leaf morphology, habit, and aroma of this accession differ significantly from that of other $O$. basilicum species and suggest the need for taxonomical reevaluation. This accession can be considered a potential source of resistance; however, its value as downy mildew-resistant breeding material needs to be determined by its capacity to cross-pollinate with a sweet basil $(O$. basilicum). In PI 296391 and PI 652054, decreased disease incidence occurred at the first true leaf pair (Table 3), demonstrating the same trend that was observed in SD.

Results of this study demonstrate that evaluation of basil cotyledons for resistance to downy mildew under controlled conditions is effective in predicting mature plant resistance. $P$. belbahrii is an obligate biotroph requiring live host tissue to survive. Thus, controlled conditions are necessary to provide an environment which ensures consistent disease pressure while maintaining physiological function and growth in the host. This study describes a two-chamber system to optimize screening conditions and which allows for accurate, reliable identification of promising genotypes. The cotyledon growth stage can be reached 7 to $10 \mathrm{~d}$ after sowing and requires a fraction of the space occupied by mature plants. In the second experiment of this study, a total of 608 seedlings, consisting of 36 USDA accessions and a control (DG), were simultaneously evaluated. An experiment of this magnitude would require extensive labor and materials if conducted as a field study.

Greenhouse screening allows for yearround evaluation and avoids the costly evaluation of susceptible genotypes in the field. Screening at the cotyledon growth stage will 
Table 3. Variation of basil response to basil downy mildew in a selection of USDA-NPGS Ocimum basilicum accessions at the cotyledon stage and first true leaf $10 \mathrm{~d}$ post-inoculation (DPI).

\begin{tabular}{|c|c|c|c|c|}
\hline \multirow[b]{2}{*}{ Accession number } & \multirow[b]{2}{*}{ Origin } & \multirow[b]{2}{*}{ USDA Accession } & \multicolumn{2}{|c|}{ Disease incidence ${ }^{z}$} \\
\hline & & & Cotyledon & First true leaf pair \\
\hline 1 & Maryland, USA & PI 652053 & 0.00 & 0.00 \\
\hline 2 & Kars, Turkey & PI 172996 & 0.00 & 0.00 \\
\hline 3 & Kars, Turkey & PI 172997 & 0.00 & 0.00 \\
\hline 4 & Van, Turkey & PI 172998 & 0.06 & 0.06 \\
\hline 5 & Iran & PI 296391 & 0.44 & 0.31 \\
\hline 6 & New Mexico, USA & PI 652054 & 0.69 & 0.44 \\
\hline 7 & Esfahan, Iran & PI 253157 & 1.00 & 1.00 \\
\hline 8 & Macedonia & PI 368700 & 1.00 & 1.00 \\
\hline 9 & Iran & PI 296390 & 1.00 & 1.00 \\
\hline 10 & Greece & PI 263870 & 1.00 & 1.00 \\
\hline 11 & Macedonia & PI 358468 & 1.00 & 1.00 \\
\hline 12 & Tokat, Turkey & PI 176646 & 1.00 & 1.00 \\
\hline 13 & Maryland, USA & PI 141196 & 1.00 & 1.00 \\
\hline 14 & Izmir, Turkey & PI 170579 & 1.00 & 1.00 \\
\hline 15 & Canakkale, Turkey & PI 170581 & 1.00 & 1.00 \\
\hline 16 & Canakkale, Turkey & PI 175793 & 1.00 & 1.00 \\
\hline 17 & Maras, Turkey & PI 182246 & 1.00 & 1.00 \\
\hline 18 & Ethiopia & PI 197442 & 1.00 & 1.00 \\
\hline 19 & Macedonia & PI 358463 & 1.00 & 1.00 \\
\hline 20 & Macedonia & PI 358464 & 1.00 & 1.00 \\
\hline 21 & Macedonia & PI 358465 & 1.00 & 1.00 \\
\hline 22 & Macedonia & PI 358466 & 1.00 & 1.00 \\
\hline 23 & Macedonia & PI 358467 & 1.00 & 1.00 \\
\hline 24 & Macedonia & PI 358469 & 1.00 & 1.00 \\
\hline 25 & Macedonia & PI 358470 & 1.00 & 1.00 \\
\hline 26 & Macedonia & PI 358471 & 1.00 & 1.00 \\
\hline 27 & Macedonia & PI 368697 & 1.00 & 1.00 \\
\hline 28 & Macedonia & PI 368699 & 1.00 & 1.00 \\
\hline 29 & Macedonia & PI 379412 & 1.00 & 1.00 \\
\hline 30 & Maryland, USA & PI 414193 & 1.00 & 1.00 \\
\hline 31 & Maryland, USA & PI 414194 & 1.00 & 1.00 \\
\hline 32 & Maryland, USA & PI 414195 & 1.00 & 1.00 \\
\hline 33 & Maryland, USA & PI 414197 & 1.00 & 1.00 \\
\hline 34 & Pennsylvania, USA & PI 601365 & 1.00 & 1.00 \\
\hline 35 & Veneto, Italy & PI 652065 & 1.00 & 1.00 \\
\hline 36 & California, USA & PI 652071 & 1.00 & 1.00 \\
\hline
\end{tabular}


resistant and $0.00=$ susceptible.

alleviate the demand on time and resources required to screen many genotypes such as in segregating breeding populations (i.e., F2 or backcross). This approach will greatly accelerate basil downy mildew breeding programs by providing an accurate, low-cost, and rapid assessment of susceptibility or resistance to BDM.

\section{Literature Cited}

Belbahri, L., G. Calmin, J. Pawlowski, and F. Lefort. 2005. Phylogenetic analysis and real time PCR detection of a presumably undescribed Peronospora species on sweet basil and sage. Mycol. Res. 109:1276-1287.

Blomquist, C.L., S. Rooney-Latham, and P.A. Nolan. 2009. First report of downy mildew caused by a Peronospora sp. in San Diego County, California. Plant Dis. 93:968.

Carovic-Stanko, K., Z. Liber, V. Besendorf, B. Javornik, B. Bohanec, I. Kolak, and Z. Satovic. 2010. Genetic relations among basil taxa (Ocimum L.) based on molecular markers, nuclear DNA content, and chromosome number. Plant Syst. Evol. 285:13-22.

Coelho, P.S., L. Valerio, and A.A. Monteiro. 2009. Leaf position, leaf age and plant age affect expression of downy mildew resistance in Brassica oleracea. Eur. J. Plant Pathol. 123:179-188.

Cohen, Y., M. Vaknin, Y. Ben-naim, A.E. Rubin, D. Silvermn, S. Bitton, and U. Adler. 2013.
First report of the occurrence and resistance to mefenoxam of Peronospora belbahrii, causal agent of downy mildew of basil (Ocimum basilicum) in Israel. Plant Dis. 97:692.1.

De Masi, L., P. Siviero, C. Esposito, D. Castaldo, F. Siano, and B. Laratta. 2006. Assessment of agronomic, chemical and genetic variability in common basil (Ocimum basilicum L.). Eur. Food Res. Technol. 223:273-281.

Dickson, M.H. and R. Petzoldt. 1993. Plant age and isolate source affect expression of downy mildew resistance in broccoli. HortScience 28:730-731.

Farahani-Kofoet, R.D., P. Römer, and R. Grosch. 2012. Systemic spread of downy mildew in basil plants and detection of the pathogen in seed and plant samples. Mycol. Prog. 11:961966.

Garibaldi, A., D. Bertetti, and M.L. Gullino. 2007. Effect of leaf wetness duration and temperature on infection of downy mildew (Peronospora sp.) of basil. J. Plant Dis. Prot. 114:6-8.

Garibaldi, A., A. Minuto, D. Bertetti, and M.L. Gullino. 2004a. Seed transmission of Peronospora sp. of basil. J. Plant Dis. Prot. 111:465-469.

Garibaldi, A., A. Minuto, G. Minuto, and M.L. Gullino. 2004b. First report of downy mildew on basil (Ocimum basilicum) in Italy. Plant Dis. 89:312.

Gilardi, G., S. Demarchi, A. Garibaldi, and M.L. Gullino. 2013. Management of downy mildew of sweet basil (Ocimum basilicum) caused by Peronospora belbahrii by means of resistance inducers, fungicides, biocontrol agents and natural products. Phytoparasitica 41:59-72.
Homa, K., W.P. Barney, D.L. Ward, C.A. Wyenandt, and J.E. Simon. 2014. Evaluation of fungicides for the control of Peronospora belbahrii on sweet basil in New Jersey. Plant Dis. (First Look). 8 Jul. 2014. <http://dx.doi.org/10.1094/PDIS-0214-0200-RE>

Jensen, B.D., J. Hockenhull, and L. Munk. 1999. Seedling and adult plant resistance to downy mildew (Peronospora parasitica) in cauliflower (Brassica oleracea convar. Botrytis var botrytis). Plant Pathol. 18:604-612.

Kanetic, L. and A. Vasiliou. 2014. First report of downy mildew caused by Peronospora belbahrii on sweet basil (Ocimum basilicum) in Cyprus. Plant Dis. 98:283.

Kim, Y.J., B.K. Hwang, and K.W. Park. 1989. Expression of age-related resistance in pepper plants infected with Phytophthora capsici. Plant Dis. 73:745-747.

Koroch, A.R., T.S. Villani, R.M. Pyne, and J.E. Simon. 2013. Rapid staining method to detect and identify downy mildew (Peronospora belbahrii) in basil. Applications in Plant Sciences 1:1300032.

Koroch, A.R., W. Wang, T.P. Michael, N. Dudai, J.E. Simon, and F.C. Belanger. 2010. Estimation of nuclear DNA content of cultivated Ocimum species by using flow cytometry. Isr. J. Plant Sci. 59:183-189.

Leckie, D., D. Astley, I.R. Crute, P.R. Ellis, D.A.C. Pink, I. Boukema, A.A. Monteiro, and S. Dias. 1996. The location and exploitation of genes for pest and disease resistance in European gene bank collections of horticultural Brassicas. Acta Hort. 407:95-101.

Martinez-de la Parte, E., L. Perez-Vicente, B. Bernal, and D. Garcia. 2009. First report of Peronospora sp. on sweet basil (Ocimum basilicum) in Cuba. New Disease Reports 20:18.

McDowell, J.M., S.G. Williams, N.T. Funderburg, T. Eulgem, and J.L. Dangl. 2005. Genetic analysis of developmentally regulated resistance to downy mildew (Hyaloperonospora parasitica) in Arabidopsis thaliana. Mol. Plant Microbe Interact. 18:1226-1234.

McGrath, M.T., A. Wyenandt, and J.E. Simon. 2010. Downy mildew wars: A monitoring program can help growers determine if the basil downy mildew pathogen is present in their area. Growing Produce: 10. 8 Feb. 2010. <http:// www.growingproduce.com/uncategorized/downymildew-wars/>.

McLeod, A., S. Coertze, and L. Mostert. 2006. First report of a Peronospora species on sweet basil in South Africa. Plant Dis. 90:1115.

Mersha, Z., S. Zhang, and R. Raid. 2012. Evaluation of systemic acquired resistance inducers for control of downy mildew on basil. Crop Prot. 40:83-90.

Monteiro, A.A., P.S. Coelho, K. Bahcevandziev, and L. Valerio. 2005. Inheritance of downy mildew at cotyledon and adult-plant stages in 'Couve Algarvia' (Brassica oleracea var. tronchuda). Euphytica 141:85-92.

Nagy, G. and A. Horvath. 2011. Occurrence of downy mildew caused by Peronospora belbahrii on sweet basil in Hungary. Plant Dis. 95:1034.

Paton, A. and E. Putievsky. 1996. Taxonomic problems and cytotaxonomic relationships between and within varieties of Ocimum basilicum and related species (Labiatae). Kew Bull. 51:509-524.

Putievsky, E. and B. Galambosi. 1999. Production systems of sweet basil, p. 39-66. In: Hiltunen, R. and Y. Holm (eds.). Basil: The genus Ocimum. Harwood, Amsterdam, The Netherlands.

Putievsky, E., A. Paton, E. Lewinsohn, U. Ravid, D. Haimovich, I. Katzir, D. Saadi, and N. Dudai. 
1999. Crossability and relationship between morphological and chemical varieties of Ocimum basilicum L. J. Herbs Spices Med. Plants 6:11-24.

Roberts, P.D., R.N. Raid, P.F. Harmon, S.A. Jordan, and A.J. Palmateer. 2009. First report of downy mildew caused by a Peronospora sp. on basil in Florida and the United States. Plant Dis. 93:199.

Silue, D., N.I. Nashaat, and Y. Tirilly. 1996. Differential responses of Brassica oleracea and $B$. rapa accessions to seven isolate of Peronospora parasitica at the cotyledon stage. Plant Dis. 80:142-144.

Simon, J.E., M.R. Morales, W.B. Phippen, R.F. Vieira, and Z. Hao. 1999. Basil: A source or aroma compounds and a popular culinary and ornamental herb, p. 499-505. In: Janick, J. (ed.). Perspectives on new crops and new uses. ASHS Press, Alexandria, VA.

Simon, J.E., J. Quinn, and R.G. Murray. 1990. Basil: A source of essential oils, p. 484-489. In: Janick, J. and J.E. Simon (eds.). Advances in new crops. Timber Press, Portland, OR.

Thines, M., S. Telle, S. Ploch, and F. Runge. 2009. Identity of the downy mildew pathogens of basil, coleus, and sage with implications for quarantine measures. Mycol. Res. 113:532-540.

Vieira, R.F., P. Goldsbrough, and J.E. Simon. 2003. Genetic diversity of basil (Ocimum spp.) based on RAPD markers. J. Amer. Soc. Hort. Sci. 128:94-99.

Vieira, R.F. and J.E. Simon. 2006. Chemical characterization of basil (Ocimum spp.) based on aromatic volatiles. Flavour Frag. J. 21:214-221.

Wang, M., M.W. Farnham, and C.E. Thomas. 2000. Phenotypic variation for downy mildew resistance among inbred broccoli. HortScience 35:925-929.

Wyenandt, C.A., J.E. Simon, M.T. McGrath, and D.L. Ward. 2010. Susceptibility of Basil cultivars and breeding lines to downy mildew (Peronospora belbahrii). HortScience 45:14161419 . 